\title{
Les transformations des registres d'action de TÜSİAD dans les années 1990 : le rôle de l'enjeu européen
}

Changes in TÜSIAD Repertoire of contention in the 1990s: the role of the European challenge

\section{Ozan Serdaroğlu}

\section{(2) OpenEdition \\ Journals}

\section{Édition électronique}

URL : http://journals.openedition.org/ejts/3837

DOI : 10.4000/ejts.3837

ISSN : $1773-0546$

\section{Éditeur}

EJTS

\section{Référence électronique}

Ozan Serdaroğlu, «Les transformations des registres d'action de TÜSIAD dans les années 1990 : le

rôle de l'enjeu européen », European Journal of Turkish Studies [En ligne], 9 | 2009, mis en ligne le 24 mars 2015, consulté le 16 février 2020. URL : http://journals.openedition.org/ejts/3837 ; DOI :

10.4000/ejts.3837

Ce document a été généré automatiquement le 16 février 2020.

(C) Some rights reserved / Creative Commons license 


\title{
Les transformations des registres d'action de TÜsİAD dans les années 1990 : le rôle de l'enjeu européen
}

\author{
Changes in TÜSIAD Repertoire of contention in the 1990s: the role of the \\ European challenge
}

Ozan Serdaroğlu

1 L'association TÜSİAD (Türkiye Sanayicileri ve Isşadamları Derneği [Association des industriels et hommes d'affaires de Turquie]) a été fondée en 1971 par douze entrepreneurs de Turquie souhaitant se démarquer de la grande majorité des entrepreneurs qui étaient jusque-là réunis sous l'égide de deux grandes organisations. La première, Türkiye Odalar ve Borsalar Birliği (ТОВВ [Union des chambres et des bourses de Turquie]) était une organisation publique rassemblant, via une adhésion obligatoire, les chambres de commerce et d'industrie de toutes les provinces turques. L'autre organisation, Türkiye İşveren Sendikaları Konfederasyonu (TísK [Confédération des Syndicats des Employeurs de Turquie]) avait été fondée en 1963 avec l'objectif de promouvoir une identité de classe et fonctionnait selon le principe d'adhésion volontaire. TISKK représentait la grande majorité des entrepreneurs turcs ayant un chiffre d'affaires modeste, et son objectif principal était de "mener une lutte sévère » contre le mouvement ouvrier et les idées de gauche, devenus plus influents après la constitution de 1961.

2 Pour leur part, les fondateurs de TÜsİAD voulaient avant tout mettre en œuvre une action non pas défensive, mais stratégique, en vue de promouvoir l'entreprenariat privé en Turquie. Dans un contexte du renforcement de certains clivages en Turquie dans les années 1960 et 1970 (ouvrier-patron / gauche-droite), ils souhaitaient promouvoir la respectabilité sociale de l'entreprenariat privé et contribuer au développement de l'économie de marché. La stratégie de TÜSİAD n'était pas tant de défendre les intérêts de ses membres face aux mouvements sociaux que de se mobiliser autour des questions de politique économique et industrielle. L'association a poursuivi cette stratégie jusqu'au début des années 1990, en publiant des études et rapports sur 
l'économie turque et en proposant des politiques publiques répondant aux besoins de l'économie de marché.

3 Depuis l'apparition d'une perspective concrète d'intégration de la Turquie à l'Union européenne, TÜSİAD ne cantonne plus son action aux questions en rapport direct avec l'entreprenariat, mais promeut également des réformes sociales et politiques, concernant les droits culturels des minorités, la liberté de pensée et la liberté de la presse, la diminution du rôle politique de l'armée, l'éducation nationale et le système judiciaire ${ }^{1}$. Elle s'engage aussi à promouvoir les relations de la Turquie avec l'Occident. Le président du Haut Conseil consultatif ${ }^{2}$ [Yüksek İstişare Konseyi], M.Mustafa Koç, a formellement déclaré que l'objectif principal de l'association était désormais d'arrimer la Turquie à l'UE (TÜSİAD 2009)3.

On peut considérer cette transformation comme un processus de politisation "qui relève (...) des tentatives de dépassement des limites assignées par la sectorisation à certains types d'activités " (Lagroye 2003: $365-366)^{4}$, alors que la sectorisation, la différenciation et la séparation fonctionnelle faisaient apparaitre nettement les frontières de la catégorie sociale constituée par les membres de TÜSİAD. Au travers d'un processus de politisation, l'association a accru sa capacité politique en dépassant les limites habituelles et institutionnalisés du cadre interprétatif de ses prises de parole, et en transmettant de nouvelles demandes, ne concernant pas exclusivement le champ économique, à des élites politiques.

Dans cet article, nous tenterons d'analyser, étape par étape, ce processus de «transgression de la différenciation des espaces d'activités et de la séparation fonctionnelle des ordres d'activités" (Lagroye $2003: 361)^{5}$. La perspective d'une intégration européenne apparait comme un facteur déterminant du processus de transgression et d'évolution de l'association. C'est pourquoi nous insisterons plus spécifiquement sur son importance, bien que l'on ne puisse la considérer comme la cause unique du passage au politique.

La première partie de l'article se focalise sur la période entre 1985-1990, lorsque TÜSİAD se tourne progressivement vers l'Occident avec la montée en puissance des entrepreneurs de la "deuxième génération ». Pendant cette période d'orientation à l'«Ouest", l'association perçoit les marchés européens comme une nouvelle opportunité économique et publie des rapports soutenant l'idée d'intégration avec eux.

La deuxième partie est consacrée aux années 1990, alors que l'association diversifie ses domaines d'activités afin de s'adapter aux nouveaux enjeux des relations euro-turques. C'est pendant cette période qu'elle commence à s'intéresser aux réformes politiques en Turquie et aux liens diplomatiques tissés entre l'Union européenne et la Turquie.

6 La troisième partie est consacrée aux conséquences pratiques des changements par lesquels est passée l'association depuis le milieu des années 1980. Notre objectif sera de montrer comment elle a su devenir partie prenante légitime des relations euroturques, agissant comme un acteur politique capable de transmettre des demandes de réformes profondes aux élites politiques turques et à l'espace public turc. 


\section{La constitution de l'Europe comme nouvel horizon d'action}

7 Cette première partie vise à montrer comment le rapport à l'Europe est devenu, à partir de 1986, un élément essentiel à la fois de l'identité et de l'agenda de TÜSIAD. Les fondateurs de TÜSİAD avaient pour point commun de compter parmi les plus grands entrepreneurs du pays, mais ils menaient leurs activités économiques dans différents secteurs. Vehbi Koç et Sakıp Sabancı possédaient les deux plus grands groupes industriels du pays. Leurs activités, comme celles du groupe de Selçuk Yaşar, se concentraient dans les secteurs de l'industrie, des services et de la finance. Les groupes de Raşit Özsaruhan et de Melih Özakat étaient spécialisés dans la métallurgie et l'automobile. İbrahim Bodur était à la tête d'un groupe spécialisé dans la production de céramique tandis qu'Osman Boyner était implanté dans le secteur du textile. Nejat Eczacıbaşı, quant à lui, possédait le plus grand groupe de pharmacologie du pays. Plus que des motivations sectorielles, l'objectif des fondateurs de l'association était de valoriser la catégorie sociale de "grands entrepreneurs». Soutenus pendant des décennies par l'État, ces grands entrepreneurs se pliaient à un corporatisme autoritaire (imposé par l'État à travers des organisations comme TOBB) et à des rapports clientélistes. Par exemple, pendant les années 1950, les plus importants entrepreneurs de Turquie tels que Vehbi Koç étaient obligés de devenir membres du parti au pouvoir et de le soutenir financièrement à travers des dons. Quand le parti kémaliste CHP [Cumhuriyet Halk Partisi] est revenu au pouvoir en 1961, certains grands entrepreneurs (Vehbi Koç et Sakıp Sabancı) se sont mobilisés directement auprès du Premier ministre pour tenter d'atténuer les effets des politiques planificatrices ${ }^{6}$.

8 La création de TÜSİAD avait pour objectif la constitution d'une véritable identité collective de grands entrepreneurs turcs et la défense de cette identité. C'est pour préserver une homogénéité que l'adhésion à l'association était difficile et basée sur le principe de sélection ${ }^{7}$. Dans un contexte d'instabilité économique et d'agitation sociale, TÜSIAD entendait contribuer à la constitution d'un environnement social et économique plus stable, propice à l'activité économique capitaliste dont la respectabilité devait être promue. Mise à part une campagne de presse, en 1979, contre le gouvernement Ecevit qui refusait de ratifier un accord de crédits avec les fonds monétaires et perpétuait, selon les membres de TÜSİAD, une approche politique populiste de l'économie ${ }^{8}$, la principale activité de l'association a longtemps été de publier des études et des rapports sur des questions économiques. Grâce aux positions particulièrement influentes des membres de l'association, ces études étaient transmises aux autres acteurs économiques, ainsi qu'aux pouvoirs publics turcs.

\section{Le rajeunissement de TÜSIAD au profit d'une génération tournée vers l'occident}

9 Dans les années 1980, TÜSIAD est devenue un pôle d'attraction pour de nombreux entrepreneurs; elle s'est développée jusqu'à compter 250 membres. Cette augmentation des adhérents a entraîné une perte d'homogénéité du groupe - en termes à la fois de profils et de motivations -, ainsi que l'apparition de rivalités entre les cadres dirigeants. L'arrivée dans les rangs de TÜSIAD de managers non propriétaires des groupes qu'ils dirigeaient a bouleversé l'équilibre interne de la structure. Les 
entrepreneurs propriétaires, fondateurs de l'organisation, ont dû partager le pouvoir et se sont heurtés à une profonde divergence d'approche, les nouveaux venus rejetant les anciens rapports clientélistes et le rôle jusque-là accordé aux gouvernements dans la vie économique. Ces divergences ont menacé le fonctionnement de l'association, les « anciens » entravant la marge de manœuvre de la nouvelle génération de managers, veillant à ce qu'en tant que représentants de l'association, ils ne commettent aucun impair vis-à-vis des représentants de l'État. Ils étaient d'ailleurs soutenus dans cette approche par les élites politiques qui considéraient elles-aussi les grands entrepreneurs propriétaires comme seuls interlocuteurs dans le domaine économique et industriel ${ }^{9}$.

10 Même si les capacités de décision de ses cadres professionnels étaient appréciées dans les entreprises où ils travaillaient et leur habileté à prendre des décisions entrepreneuriales, reconnue, les membres fondateurs et les anciens membres critiquaient leur prépondérance au sein du conseil d'administration. Ils considéraient en effet qu'ils n'étaient pas en mesure de représenter les entrepreneurs d'un point de vue identitaire, ce qui constituait pourtant l'objectif premier de TÜSİAD. Selon eux, les "professionnels » pouvaient contribuer à l'action de TÜSİAD, mais ne devaient en aucun cas l'orienter; l'association devait rester dirigée par des entrepreneurs propriétaires ${ }^{10}$.

11 Les réticences de ces membres prépondérants ont été renforcées en 1985 lorsque la liste de M. Kocatopçu a gagné les élections du conseil d'administration. M. Kocatopçu était un PDG et non un entrepreneur propriétaire ${ }^{11}$ et son conseil d'administration a d'ailleurs été surnommé l'« équipe B de TÜSİAD $»^{12}$, car il comprenait majoritairement des directeurs et des membres des comités de direction d'entreprises.

12 Les grands entrepreneurs ont très vite réagi et ont gagné les élections de 1986, avec la liste de Sakıp Sabanci. En effet, S. Sabanc1 ${ }^{13}$ a choisi de contrer ces managers professionnels en promouvant de jeunes membres au sein des instances dirigeantes de l'association. Ces « jeunes » n'étaient autres que les fils des grands entrepreneurs de la première génération. Sur les dix membres qui composent son conseil d'administration, on trouve trois jeunes entrepreneurs propriétaires de la deuxième génération (qui seront trois futurs «jeunes présidents»). Parmi eux, Bülent Eczacıbaşı explique explique à propos de sa place au sein de l'association : «L'année 1986 était considérée comme une année de transition pour TÜSİAD. Sakıp Sabancı avait accepté la présidence $\mathrm{du}$ conseil d'administration pour un an. Il souhaitait installer aux postes d'administration un groupe d'hommes d'affaires jeunes. Il m'avait dit qu'il voulait me donner une place dans sa liste, avec Ömer Dinçkök et Cem Boyner (deux autres futurs présidents de TÜSİAD pendant la deuxième moitié des années 1980). (...) Sakıp Bey a dit ouvertement: "n'attendez pas de sacrifices de ma part si je ne vois pas la présence de jeunes au tour de moi" $»^{14}$.

13 Or, cette nouvelle génération avait pour particularité d'être largement acquise aux référents occidentaux. Bülent Eczacıbaşı (né en 1949), Ömer Dinçkök (né en 1948) et Cem Boyner (né en 1955) ont connu des socialisations et formations similaires. Bülent Eczacıbaşı est diplômé du lycée allemand d'Istanbul. Il a poursuivi ses études universitaires à l'université de Londres avant d'obtenir son master en chimie au Massachusetts Institute of Technology. Ömer Dinçkök est diplômé d'un lycée américain à Istanbul, le Robert College, et y a poursuivi ses études, au département de gestion et d'économie d'entreprise de son école supérieure. Cem Boyner, également diplômé du Robert College, a poursuivi ses études à l'université de Boğaziçi, qui n'était autre que la 
section «école supérieure » du Robert College après son transfert à l'État turc. Avec une telle formation, tournée vers l'Occident, les trois entrepreneurs ont été éduqués au sein de milieux intellectuels échappant en partie à certains formatages cognitifs imposés par les institutions publiques éducatives et culturelles turques ${ }^{15}$. Leurs modes de pensée et de vie, contrairement à ceux de leurs pères, ont été façonnés par la culture occidentale. Ces codes et références occidentaux contribueront au rapprochement avec la bourgeoisie occidentale.

\section{Les marchés européens : une opportunité économique}

Cette «occidentalisation» de l'équipe dirigeante de TÜSIAD s'est combinée à l'émergence des marchés européens comme nouvelle opportunité économique pour la Turquie. Dans un climat de libéralisme économique accru, l'adoption des décisions du 24 janvier 1979, en facilitant l'exportation industrielle (Boratav 2003: 149) ${ }^{16}$, avait généralement bénéficié aux membres de TÜSIAD. Les meilleurs débouchés de l'industrie turque étaient les pays du Moyen-Orient ${ }^{17}$.

En 1985, pour la première fois depuis 1979, les exportations industrielles turques ont diminué, du fait notamment de la baisse du pouvoir d'achat au Moyen-Orient (provenant de la baisse des prix de pétrole) et des effets dévastateurs de la guerre entre l'Iran et l'Irak. En réaction, l'association a d'abord effectué une mise à jour de ses priorités: plutôt que de s'en prendre au rôle joué par l'État dans le domaine économique, la nouvelle direction a considéré qu'il y avait urgence à réformer la «mentalité générale » dominant la vie économique en Turquie. Dans son rapport de 1986 sur le commerce extérieur ${ }^{18}$, l'association affirme que la l'industrie turque doit développer son ouverture aux marchés plus développés, ne pas avoir peur de la compétition et accroître la qualité et la quantité de sa production. L'ouverture aux marchés européens se justifie du fait de leur richesse et de leur stabilité. De plus, l'association considère qu'à la différence des marchés américains ou d'Extrême-Orient (comme le Japon), il existe une opportunité concrète d'intégration à la Communauté économique européenne (CEE). Avec la signature de l'accord d'association en 1963, la CEE et la Turquie avaient prévu la signature d'une union douanière et s'étaient engagées à diminuer leurs droits de douane. Cependant, depuis le coup d'État de 1980 en Turquie, les relations euro-turques étaient gelées ${ }^{19}$. L'association va donc plaider pour leur reprise, proposant notamment, dans son rapport de 1986, que le gouvernement turc se batte contre les mesures de protection dont bénéficiaient les industries européennes..

\section{L'intégration européenne : la nouvelle priorité de TÜSIAD}

16 L'ouverture aux marchés européens est apparue, dans la deuxième partie des années quatre-vingt, comme la priorité de TÜSIAD. L'objectif ultime n'était plus d'œuvrer pour promouvoir l'environnement direct de l'entreprenariat privé mais d'éliminer les obstacles à l'intégration aux marchés européens. Pour cela, l'association a doublé ses activités classiques - publication de rapports ${ }^{20}$ et contacts avec les acteurs économiques et politiques turcs - d'un nouveau type d'action : la diplomatie internationale.

17 L'association a pris contact non seulement avec les milieux d'affaires européens mais également avec les plus hautes autorités politiques et sectorielles. Ainsi, en avril 1986, 
des délégations de l'association ont rencontré les ministres de l'Economie et de l'Industrie français, le Conseil des employeurs nationaux des pays membres de l'Union, ainsi que certains responsables européens en charge des relations avec la Turquie. L'objectif était de démontrer la détermination de l'association à œuvrer pour l'intégration de la Turquie dans la Communauté économique européenne. Toujours en avril 1986, Vehbi Koç a clairement exprimé que la Turquie souhaitait prendre part à la construction européenne. Et le président Sakıp Sabancı a appelé les élites politiques européennes à prendre en compte cette détermination :

«Que vous le souhaitiez ou non, La Turquie va devenir un membre de la CEE !» (Birand $1996: 397)^{21}$.

Suite à la demande d'adhésion de la Turquie à la CEE en $1987^{22}$, TÜSİAD a entamé un processus de "supranationalisation", c'est-à-dire d'«institutionnalisation des liens entre associations et groupes d'intérêts nationaux au niveau communautaire" (Grossman 2002: 45)23. L'association a en effet adhéré en 1988 à l'UNICE (Union des confédérations de l'industrie et des employeurs d'Europe, devenue en 2007 Business Europe). Cette confédération des organisations entrepreneuriales européennes était considérée par les institutions européennes comme un "partenaire social » de la CEE dans le domaine de la politique sociale. De ce fait, elle était formellement impliquée dans le processus de décision européen, pour représenter les intérêts supranationaux de ses membres. Avec cette adhésion, TÜSİAD participait à un nouveau réseau d'interaction entre élites privées et publiques européennes. Ce rapprochement multiforme avec l'Europe est essentiel pour saisir les transformations qui vont par la suite affecter l'organisation.

\section{Un élargissement des secteurs d'intérêt de l'association : le passage au politique}

Dans les années quatre-vingt-dix, les domaines d'intérêt de l'organisation vont s'élargir pour intégrer, au-delà des perspectives économiques, une dimension politique. L'association va peu à peu proposer des réformes qui ne visent plus seulement à transformer le secteur économique, mais également à réformer le système politique et social sur le modèle des démocraties occidentales. Cet élargissement des domaines d'intérêt va de pair avec une transformation des modes d'action de TÜSİAD: l'association ne se contente plus de faire des propositions mais cherche à institutionnaliser un dialogue permanent avec les gouvernants sur les questions qui la préoccupent. Les conséquences de ces transformations se font sentir au-delà du contexte domestique. Les institutions européennes vont rapidement considérer TÜSIAD comme l'un de leurs meilleurs partenaires turcs dans la gestion des relations complexes entre l'Union européenne et la Turquie. Dès lors, l'association s'institue comme une interface entre institutions européennes et turques.

\section{Les prémices d'une nouvelle ère}

21 A partir de 1989, la Commission européenne met l'accent sur une possible adhésion de la Turquie, sous certaines conditions, et propose le passage à l'union douanière ${ }^{24}$. Cette ouverture exigeait une transformation considérable du cadre juridique de l'activité économique, notamment la suppression de certaines régulations commerciales 
constituant des distorsions à la concurrence ou des barrières à la libre circulation des biens, des services, des personnes et des capitaux ${ }^{25}$. De telles exigences allaient dans le sens des attentes de la direction de TÜSİAD, alors que l'Union douanière devenait l'objectif de l'organisation. Selon Cem Duna, membre prépondérant de TÜSİAD « Avec l'ouverture de la perspective d'union douanière, la compétitivité est devenue la règle pour les entrepreneurs turcs, et aussi pour les institutions publiques $»^{26}$.

D'après Bülent Eczacıbaşı (l'un des jeunes entrepreneurs de la deuxième génération élu en 1986, qui deviendra président du conseil d'administration en 1991), les acteurs économiques ont besoin d'une sécurité assurée par l'existence de règles concrètes déterminant les principes d'intervention gouvernementale dans l'économie. Le manque de sécurité et de confiance dans les gouvernants a donc amené TÜSİAD à souhaiter des garanties constitutionnelles. Bülent Eczacıbaşı appelait cette approche «économie constitutionnelle »: "Peut-être le concept d'économie constitutionnelle n'apportera pas au début des contributions concrètes et applicables à la Turquie. En revanche, nous voudrions exprimer notre vision selon laquelle la Turquie ne doit pas se fermer aux approches qui n'ont pas encore été suffisamment expérimentées (...) L'économie turque a besoin d'une constitution limitant la volonté des hommes politiques dans le domaine économique pour encadrer leurs décisions en fonction de certains principes ${ }^{27}$ (Eczacıbaş1 1992 : 15-16).

En 1992, TÜSIAD transgresse les frontières du secteur économique pour s'intéresser à un projet de constitution démocratique. Pour ce faire, l'association crée un comité composé de six professeurs et trois maîtres de conférences en droit et sciences politiques, qui rédigent une étude nommée « Pour une nouvelle constitution » (TÜSİAD 1992) ${ }^{28}$. Cette étude préconise certaines réformes pour la promotion de la démocratie et l'amélioration de la situation des droits de l'homme en Turquie : rattachement du chef d'État-major au ministère de la Sécurité nationale ; reconnaissance à chacun du droit de parler librement sa langue maternelle et de fonder des radios et télévisions; diminution de la durée des gardes à vue; indépendance de l'«Institut de la langue turque » et de l'« Institut de l'histoire turque ${ }^{29}$.

Il est pertinent de rappeler le contexte politique domestique et international de cette initiative. D'un point de vue international, TÜSIAD a rapidement perçu le lien fort entre opportunités d'ouverture économique et respectabilité internationale de la Turquie. En décembre 1989, la réponse de la Commission européenne à la demande d'adhésion de la Turquie a clairement souligné que l'approfondissement des relations euro-turques dépendait de la promotion des droits de l'homme et de la démocratisation en Turquie ${ }^{30}$. Au début des années 1990, l'intérêt croissant de TÜSİAD pour les questions politiques et juridiques coïncide donc avec le débat sur la «démocratisation» qui mobilisait les élites politiques de Turquie.

Les questions de la "démocratisation » et de la "promotion des droits de l'homme » ont été mises à l'agenda par le gouvernement de coalition formé par le Parti social démocrate populaire SHP [Sosyal Demokrat Halkçı Parti], social-démocrate/centre gauche, et le parti de la Juste Voie DYP [Doğru Yol Partisi], libéral/centre droit, qui avait succédé au gouvernement de Turgut Özal, ANAP (Anavatan Partisi [parti de la Mère Patrie]), libéral/centre droit, en 1991. Le programme de ce gouvernement mettait particulièrement l'accent sur la situation des minorités et le besoin de représenter la pluralité sociale en politique. Par ailleurs, lors des législatives de 1991, vingt-deux députés issus du parti nationaliste kurde Demokrasi Partisi [parti de la Démocratie] ont été élus députés. La langue kurde a été officiellement reconnue la même année, par la 
volonté de Turgut Özal, désormais Président de la République. En conformité avec son programme, le gouvernement avait commencé à élaborer un "paquet de démocratisation" comprenant certains amendements à des lois et dispositions constitutionnelles. Il a également instauré un ministère des droits de l'homme. A cette période, certains détenus politiques ont obtenu une libération conditionnelle. Toutes ces initiatives, marquaient la volonté du gouvernement de réduire les obstacles à la liberté d'expression et aux droits culturels, obstacles sur lesquels le projet de nouvelle Constitution de TÜSIAD revenait largement.

Même si TÜSIAD ne s'est pas complètement approprié cette étude « Pour une nouvelle Constitution ", qui reste d'ailleurs peu connue de ses membres - dans les années qui suivirent, les questions des droits de l'homme ou de démocratie en Turquie n'ont pas été mises en avant dans les activités et les discours engageant l'association pendant quelques années et les propositions contenues dans le rapport sont avant tout celles d'universitaires intellectuels très critiques vis-à-vis de la Constitution de 1982 - ce projet met néanmoins en évidence une nouvelle tendance de l'association à aborder les problèmes politiques du pays bien au-delà des seules questions économiques. Cette tendance va ensuite se renforcer. En cela, ce projet peut être considéré comme le précurseur d'une nouvelle ère pour TÜsİAD.

\section{L'Union douanière, une étape décisive}

Sous le mandat d'Halis Komili ${ }^{31}$ qui débute en 1993, TÜSİAD donne la priorité à l'aboutissement de l'Union douanière. Pour cela, l'organisation joue pleinement la carte de l'intégration dans l'eurogroupe patronal UNICE et espère que ses partenaires européens joueront le rôle d'intermédiaires de la cause turque. C'est ce que nous confirme Hale Hatipoğlu, sous-secrétaire de TÜSİAD en charge du département des relations extérieures à la date de l'entretien :

«TÜSİAD a demandé aux autres membres de l'UNICE d'agir auprès de leurs gouvernements et des parlementaires européens pour une décision en faveur de l'union douanière, en la considérant comme une étape menant à l'adhésion de la Turquie. TÜSIAD n'avait pas de représentation permanente à Bruxelles, et nous n'avions pas de discours persuasif $»^{32}$.

En revanche, les relations entre TÜSIAD et le gouvernement turc se complexifient au moment de la négociation de l'union douanière, car les institutions turques cherchent à exclure l'association des négociations, voire à empêcher certaines de ses activités de lobbying. Sinan Ülger, un ancien diplomate turc ayant participé à ces négociations, nous explique le contexte de relations entre le gouvernement et TÜSİAD pendant cette période :

«Quand les négociations sur l'union douanière ont commencé, au lieu de coopérer avec TÜSİAD, le gouvernement a poursuivi des stratégies d'exclusion absolue des entrepreneurs. Les représentants turcs avaient le souci d'empêcher que TÜSİAD ne sabote l'affaire en utilisant les canaux diplomatiques, et qu'elle n'influence le déroulement des négociations selon ses propres intérêts. Le gouvernement et le ministère des Affaires étrangères pensaient mener les négociations comme ils le souhaitaient, le plus vite possible, puis ensuite négocier avec TÜSİAD. C'était ainsi qu'il en a été pour les négociations techniques " ${ }^{33}$. dossier a été transmis au Parlement européen en 1995 pour avis conforme. Le ministère 
des Affaires étrangères, conscient de la coopération entre TÜSİAD et l'UNICE, a commencé à accepter de partager les responsabilités dans le domaine diplomatique. C'est ce que nous confirme Sinan Ülgen, ancien diplomate :

«Quand l'affaire est arrivée devant le Parlement européen, les relations entre TÜsİAD et les institutions publiques turques ont commencé à changer. Par exemple, notre ministère des Affaires étrangères s'est engagé à contribuer aux activités de lobbying de TÜSİAD : nous avons utilisé nos propres canaux diplomatiques pour prendre des rendez-vous entre les parlementaires européens et des représentants de TÜSİAD, et nous avons facilité leur participation à certaines réunions et activités au sein des institutions européennes. Une coopération, jamais vue jusque-là, a eu lieu. L'affaire était en train de se politiser et nous avons vu à ce moment-là qu'il était dans notre intérêt de jouer le jeu avec TÜSİAD " $^{34}$.

31 Les responsables turcs ont donc considéré de leur intérêt de mettre en avant des organisations de la "société civile» turque, sensibles notamment aux questions des droits de l'homme, pour promouvoir la Turquie auprès des parlementaires et plus généralement auprès des institutions communautaires.

Au-delà de l'évolution des rapports avec le gouvernement, il n'y a pas véritablement eu de transformation du message de TÜSİAD qui consistait exclusivement à défendre l'idée d'union douanière.

«Quelquefois, certains membres de TÜSİAD se sont déplacés à Bruxelles pour suivre le processus de négociation et rencontrer les représentants des institutions européennes. Ils leur ont simplement dit "dites oui à l'union douanière avec la Turquie". Mais pour quelles raisons? Pourquoi les parlementaires européens diraient "oui"? Il fallait avoir de bonnes réponses et les expliquer aux hommes politiques européens $»^{35}$. Hale Hatipoğlu souligne ainsi les limites du lobbying mené par TÜsİAD dans ce contexte.

\section{La nécessaire redéfinition d'une cause autour de la candidature européenne}

Selon le chef de l'unité «Turquie » de la direction générale de l'élargissement de la Commission européenne en 2002, Adriann Van der Meer, c'est à partir du moment où TÜSIAD a commencé à faire sien le constat d'une nécessaire démocratisation qu'elle a été prise en compte par les institutions européennes. Jusqu'à la deuxième moitié des années 1990, elle n'était pas vraiment considérée comme un acteur susceptible d'orienter les décisions des autorités européennes concernant la Turquie : «A cette époque où les problèmes politiques dominaient le climat des relations, la Commission européenne s'intéressait particulièrement aux problèmes des droits de l'homme en Turquie. Nous recueillions nos informations sur ces problèmes par l'intermédiaire d'organisations non gouvernementales comme Amnesty International, Human Rights Watch, ou l'association turque des droits de l'homme IHD [İnsan Hakları Derneği]. (...) TÜSİAD n'était pas une association ayant un rôle dans cette division du travail $»^{36}$.

Sans forcément partager cette vision des choses, les membres de TÜSİAD ont pris conscience qu'ils ne pouvaient plus se contenter de plaider en faveur de la poursuite du processus d'intégration. Dans cette relation dissymétrique, l'association se devait d'aligner ses priorités sur celles des institutions européennes pour s'assurer un minimum de reconnaissance et d'influence et d'afficher un engagement plus radical sur les questions politiques de démocratisation. Alors que TÜSIAD consacrait une partie de plus en plus importante de ses moyens et de ses ressources au projet d'intégration 
européenne, la structure de l'organisation devait aussi s'adapter de manière à contribuer durablement et efficacement à la mise en œuvre de ce processus.

La restructuration a commencé par la création de huit comités en $1995^{37}$ dont l'objectif était de se « concentrer sur les effets éventuels de l'union douanière dans leur domaine d'activité ${ }^{38}$. Les présidents de ces comités sont tous membres du conseil d'administration de l'association, ce qui indique l'importance que leur confère l'organisation. Pour soutenir les activités de ces comités, l'association a décidé en 1996 de créer de nombreux groupes de travail. Jusqu'en 1999, ces groupes de travail fonctionnaient comme des groupes ad hoc, mais à partir de la reconnaissance officielle de la candidature de la Turquie à Helsinki, ils ont été organisés de manière à prendre en compte l'« acquis communautaire ${ }^{39}$ dans leurs domaines de compétence.

La restructuration de TÜSİAD se caractérise également par la multiplication des personnes travaillant au sein de son secrétariat général ${ }^{40}$ ainsi que par la création de départements, (au sein du secrétariat) qui suivent les travaux des comités et des groupes de travail et qui élaborent les bases de leurs activités et de leur ordre du jour. L'organisation du secrétariat général est passée d'une vingtaine de salariés dans les années quatre-vingt à 60 aujourd'hui. Ils sont en permanence au service des membres de TÜSİAD, à travers les études et recherches qu'ils effectuent. Ils suivent, de façon plus générale, l'ensemble des transformations politiques et socio-économiques nationales et internationales.

37 A la suite de cette restructuration interne, l'association s'est en effet organisée à l'international. En 1996, elle a ouvert un bureau de représentation permanente à Bruxelles pour être plus présente au sein de l'UNICE, contribuer au processus technique d'harmonisation lié à l'union douanière, recueillir des informations sur les relations entre l'UE et la Turquie et prendre plus d'initiatives diplomatiques afin d'influer sur celles-ci. Les activités de cette représentation sont principalement destinées à la promotion de la candidature de la Turquie et à l'interaction avec les acteurs diplomatiques et les organisations entrepreneuriales internationales. Le bureau n'a pas pour objectif de faire des affaires; une autre organisation, «TUSIAD international » a été créée en 2001 pour développer les affaires entre les membres de TÜsİAD et les entrepreneurs étrangers.

La restructuration de TÜSİAD semble avoir accru son efficacité. La meilleure démonstration en est la multiplication des études de TÜSİAD en 1995. Alors qu'auparavant l'association publiait en moyenne neuf rapports et études par an (223 publications en 24 ans), elle a rédigé 24 rapports et études en 1995, la première année de sa restructuration, qui est également l'année de la conclusion de l'Union douanière ${ }^{41}$. Pendant la période 1995-2004, l'association a publié en moyenne 23 études par an (233 publications en 10 ans). Dans ces publications, on constate les effets du contexte de l'Union douanière. Parmi les 24 études et rapports publiés en 1995, 20 concernaient le domaine économique et les stratégies de promotion de la compétitivité et de la qualité de production nationales. Cette tendance s'est poursuivie en 1996, alors que la compétitivité et le contexte économique constituaient les priorités à l'ordre de jour de l'association.

39 L'association a publié des rapports sur le domaine économique, mais aussi politique, ce qui indique la volonté d'élargissement de ses domaines d'intérêt. Trois premiers rapports, publiés en 1995, («Vers un nouveau système électoral pour la Turquie », «L'État optimal », « Le débat sur le système électoral et le système à deux tours ») sont 
suivis en 1997 d'une étude plus revendicative réalisée par un sociologue reconnu proche des idéologies gauchistes, Bülent Tanör, intitulée "Les perspectives de démocratisation en Turquie $»^{42}$, qui marque un tournant dans les revendications de l'association et son rapport aux questions politiques.

\section{L'émergence de TÜSIAD comme éclaireur politique}

En effet, l'étude «Perspectives de démocratisation en Turquie » propose des réformes radicales du système politique et judiciaire qui visent la modification de certains fondements du régime de 1982 : la diminution du rôle politique et juridique de l'armée, la reconnaissance de la pluralité culturelle dans le pays, la libéralisation du cadre juridique des actions collectives et une réforme approfondie dans l'éducation nationale. Le rapport marque une rupture avec les études précédentes parce qu'il ne préconise plus seulement de limiter l'arbitraire des pouvoirs publics dans la politique économique ou la politique industrielle. Il s'agit d'un engagement plus profond, lié à un changement de visions et d'objectifs au sein de TÜsİAD : les membres actifs, notamment ceux présents dans l'administration de l'association et prenant part aux activités des comités et des groupes de travail, admettent que «leur activité professionnelle, au-delà de ses finalités propres, puisse être orientée, voire inspirée, par des objectifs d'ordre politique, à condition que ces derniers soient inscrits dans une vision large du politique (la démocratisation, le combat pour la justice et l'égalité de traitement des autres, l'éducation des citoyens), et non (...) dans une conception limitée et suspecte de la politique partisane » (Lagroye 2003: 366) ${ }^{43}$. La brochure élaborée par le secrétariat général de l'association après la publication du rapport indique que «en plus de la poursuite de conditions économiques stables, TÜSİAD se concentre sur la stabilité politique à travers l'amélioration des conditions démocratiques $»^{44}$. Ainsi, pour reprendre des termes de Jacques Lagroye, TÜSİAD montre que son action peut aussi avoir une "portée politique générale ", et qu'elle entend "participer à l'avènement d'une démocratie de participation, lutter contre les oppressions et injustices, et contraindre les élus à agir d'une certaine manière » (Lagroye $2003: 362-363)^{45}$. De ce fait, avec ce rapport, TÜSİAD tente de devenir un éducateur politique.

\section{Le virage politique de l'association}

41 Comme tous les rapports de l'association, ce rapport a été initié par le conseil d'administration de TÜSİAD, qui a également décidé de le publier, après consultation du Haut Conseil de consultation. Il a toutefois suscité de nombreux remous au sein de l'association. Lors du $27^{\text {e }}$ Conseil général du 23 janvier 1997, trois jours après la publication, certains membres ont critiqué son coût financier, et souligné qu'il ne reflétait pas leurs vues, mais celles de Bülent Tanör. Leur opposition a été efficace et le rapport a été enlevé du rapport d'activité de 1996. Cependant, d'anciens présidents, pionniers du changement de TÜSİAD, comme Sakip Sabanc1 et Cem Boyner, ont défendu le rapport. Le 8 avril 1997, lors de la réunion du Haut Conseil de consultation de TÜSİA, le rapport a été accepté « en conformité avec la mission de l'association », marquant une fois encore la victoire de la jeune génération.

A la suite de ce rapport, l'association a continué à publier des études sur la question de la démocratisation et des réformes juridiques. Pendant les mandats de Muharrem 
Kayhan (1997-1999), Erkut Yücaoğlu (1999-2001) et Tuncay Özilhan (2001-2004), l'association a consolidé ses contributions à la démocratisation en Turquie. Elle s'est engagée dans de nouvelles actions encourageant les interactions entre entrepreneurs, hommes politiques, parlementaires, administrateurs et experts intéressés par les problèmes démocratiques.

L'activité d'expertise de l'organisation s'est doublée d'une activité de plus en plus importante de contacts directs avec les acteurs politiques. Ces contacts sont en général le fait des groupes de travail qui se réunissent régulièrement avec les responsables politiques depuis leur création en 1995. Cependant, son opérationnalité s'est accrue suite à l'acceptation de la candidature turque en 1999, et la création du bureau d'Ankara en 2000. La fonction générale de ce bureau est de suivre les projets de loi au Parlement et le processus de prise de décision par le gouvernement. Comme celui d'Istanbul, ce bureau est composé de six salariés recrutés par TÜsİAD. Grâce à la légitimité montante de l'association, ces salariés participent à « des réunions avec les ministres et haut fonctionnaires, ils notent leurs vues, discutent avec les parlementaires et fournissent ainsi des informations que le siège d'Istanbul ne peut pas obtenir autrement $»^{46}$. La communication avec les parlementaires est donc devenue une activité essentielle. Il ne s'agit pas simplement d'informer sur les propositions de l'association, mais d'influencer l'élaboration des politiques publiques sur des questions politiques (tels que le système des partis politiques, les élections, la Grande Assemblée Nationale de Turquie et le système gouvernemental, les municipalités, l'État de droit et la justice et enfin les Droits de l'homme).

Pour exercer cette pression politique, l'association compte d'abord sur son statut (association de grands entrepreneurs, de leaders économiques qui se sont dévoués au bien de leur pays), sur la justesse de ses propositions (du point de vue scientifique et en prenant en compte le contexte, en expliquant pourquoi il est temps de procéder à des réformes) et sur la capacité de ses membres à persuader les parlementaires. C'est pour cette raison, comme l'explique Serkan Ersöz du département des affaires parlementaires de TÜSİAD, que les délégations envoyées à Ankara sont constituées de membres ayant d'importantes capacités relationnelles et communicationnelles :

«Nous préférons travailler avec les membres qui sont connus dans les milieux politiques et qui savent comment s'adresser aux hommes politiques et aux parlementaires. La question des réformes politiques est un sujet délicat, et nous avons des attentes de réformes urgentes. Quelqu'un qui est appelé à contribuer à la formulation de notre position dans ce domaine doit avoir une efficacité particulière pour persuader les responsables politiques. Il doit très bien savoir ce qu'il est possible de faire en Turquie, quand et dans quelles conditions (...) C'est pour cette raison que nous travaillons avec des membres comme Can Paker $^{47}$ et Erkut Yücaoğlu ${ }^{48} »^{49}$.

TÜSIAD passe ainsi d'un simple rôle d'expert à celui de véritable acteur politique.

\section{Une légitimité accrue au niveau européen}

Traduit en anglais et en allemand, le rapport sur les perspectives de démocratisation de 1997 est devenu une étude de référence pour les organisations internationales ${ }^{50}$ tandis que plusieurs responsables de la Commission européenne l'ont consulté sur le site internet de TÜSIAD. Sur la base du rapport intégral publié, le bureau bruxellois de TÜSIAD en a diffusé un résumé de deux pages aux institutions européennes, aux 
hommes politiques des pays membres et à de nombreux acteurs de la société civile. Cette démarche a été efficace puisque de nombreux responsables européens ont considéré ce document comme devant servir de plan d'orientation ${ }^{51}$. Ainsi, selon le chef d'unité Turquie au sein de la direction générale à l'élargissement de la Commission européenne, en poste à cette époque : « Depuis la fin des années 1990, nous considérons TÜSIAD comme l'un de nos meilleurs partenaires. L'association a publié d'importants rapports, et en même temps a réussi à débattre des réformes avec les décideurs politiques. Elle a pris une initiative remarquable pour la démocratisation. Nous serions très contents si tout le monde en Turquie partageait la vision de TÜSİAD. Evidemment ceci accélérerait le processus d'intégration de la Turquie $»^{52}$.

Grâce à un positionnement critique face aux problèmes politiques de son pays, TÜSIAD a pu acquérir une certaine légitimité au niveau européen qui lui a permis d'approfondir ses contacts avec les parlementaires européens et les eurocrates de la Commission pour discuter des réformes engagées par la Turquie. Dans le cadre d'un lobbying de grande ampleur mené en 1998-1999 (pour obtenir l'acceptation de la candidature turque) dirigé vers ses partenaires de l'UNICE et les hommes politiques de premier plan des pays membres, le discours de TÜSİAD insistait sur l'engagement pris par la Turquie sur la voie de la démocratisation et la nécessité d'encouragements européens pour poursuivre dans cette voie.

\section{Une légitimité en progression au niveau national}

L'engagement de l'organisation dans la promotion de la candidature a également accru la légitimité politique de TÜSİAD vis-à-vis des institutions publiques turques. L'État turc reconnait aujourd'hui à TÜSİAD un poids paradiplomatique, en particulier à Bruxelles: "Auparavant, les relations entre l'UE et la Turquie étaient des relations largement dominées par les volontés des acteurs étatiques. Le rôle des acteurs civils était très limité. Après l'Union douanière, ce paradigme a changé et l'État turc a vu que la coopération avec la société civile était nécessaire. (...) Il a vu qu'il devait jouer le jeu démocratique correctement mais qu'il était déficient sur ce terrain. Il a alors commencé à entrer plus facilement en relation avec la société civile, ce qui a donné de bons résultats ${ }^{53}$.

Aujourd'hui, les dirigeants de TÜSİAD savent que les institutions publiques turques sont conscientes du poids diplomatique de leur association. Elles cherchent à coopérer davantage avec TÜSİAD et demandent même son soutien diplomatique sur des questions sensibles : «Nos hommes politiques connaissent notre capacité et veulent en bénéficier dans d'autres domaines que les relations avec l'UE, par exemple, la question arménienne. Au dernier moment un ambassadeur m'appelle, le ministre des Affaires étrangères m'appelle. Ils me demandent "est-ce que vous pouvez être là dans quatre heures ?". Je dis "Oui, d'accord. Mais qu'est-ce que vous avez fait jusqu'à maintenant? Pourquoi vous vous êtes tu ?" ${ }^{54}$.

50 Malgré ces critiques vis-à-vis du régime, les acteurs étatiques reconnaissent que TÜSİAD fait preuve de "patriotisme " puisque son objectif est la défense de l'intérêt et de la candidature de la Turquie. Sa posture peut être résumée de la manière suivante : «si vous avez besoin de moi, c'est parce que je remplis une fonction que vous n'avez jamais pu remplir ». 
51 TÜSIAD est parvenue à se faire reconnaître par les officiels turcs comme un interlocuteur incontournable et légitime en dépit de son discours critique. Cela tient d'une part à la légitimité qu'elle a su acquérir au niveau européen et d'autre part au fait qu'au-delà de son discours critique, l'association se fait l'avocat de la cause nationale turque auprès des instances européennes.

\section{Conclusion}

Certains membres de TÜsİAD définissent aujourd'hui l'association comme un think tank : «Après avoir fonctionné selon des préoccupations professionnelles pendant des décennies, nous fonctionnons aujourd'hui comme un think tank faisant de la recherche sur les questions politiques du pays $»^{55}$.

53 Cette transformation de l'organisation crée des divergences entre les membres. Ceux qui sont le plus actifs ainsi que les salariés composent un groupe cohérent, rassemblé autour de leur engagement pour la transformation politique du régime. Ceux qui exercent des responsabilités dans le comité des affaires parlementaires sont ceux qui sont les plus engagés dans la démocratisation. Mais cette partie active de l'association comprend une minorité des membres de TÜSİAD : entre 1994 et 2006, trente-deux membres par an en moyenne ont participé aux activités des comités et groupes de travail, tandis que le nombre total des membres pendant cette période s'élevait à cinq cent soixante. Serkan Ersöz confirme qu'il existe une certaine division au sein de l'association :

«Etre entrepreneur n'implique pas être réformiste. Parmi nos membres il y a aussi des conservateurs et des nationalistes. Ils s'opposent à la majorité de nos activités concernant la démocratisation et les réformes politiques. Cette opposition ne résulte pas seulement de leurs liens particuliers avec l'État ou l'armée. Idéologiquement aussi, ils peuvent être contre notre engagement en faveur de la démocratisation ${ }^{56}$.

54 Quelles que soient les critiques ou les oppositions à la nouvelle orientation de l'organisation, les membres passifs ne constituent pas un groupe cohérent. Ils ne partagent pas d'objectifs ou de projet susceptible d'être construit en référent alternatif à celui du groupe actif. Les oppositions n'ont pas n'ont plus entraîné de défection de membres. En effet, l'adhésion continue à offrir de nombreux avantages. Pour les "grands entrepreneurs turcs », TÜSİAD peut servir d'instrument d'ouverture sur le monde extérieur et favoriser les opportunités économiques vers l'étranger. De plus, devenir membre de TÜSIAD signifie un accès facilité aux espaces politiques turc et européen.

\section{BIBLIOGRAPHIE}

Birand, Mehmet Ali (1996) Türkiye'nin Gümrük Birliği Macerası 1959-1996 [L'aventure d'Union douanière de la Turquie 1959-1996], Istanbul, Ad yayincllk, p. 397. 
Avis de la Commission sur la demande d'adhésion de la Turquie à la Communauté, 20 décembre 1989, SEC (89) 2290 final, http://www.ena.lu/mce.cfm, dernière consultation le 2 août 2007.

Boratav, Korkut (2003) Türkiye İktisat Tarihi 1908-2002 [Histoire économique de la Turquie 1908-2002], İmge, Ankara, p. 149.

Buğra, Ayşe (1997) Devlet ve İşadamları [État et hommes d'affaires], Istanbul, İletişim, pp. 176-197.

Dündar, Can (2006) L'archive privée de Vehbi Koç a été ouverte à la $1^{\text {me }}$ anniversaire de son décès, URL : www.candundar.com.tr Eczacıbaşı (1992) «Toplumun Katılımına Açık Bir Anayasa » [Une constitution ouverte à la participation de la société], in Görüş, Istanbul, TÜsİAD, pp. 15-16.

Grossman, Emiliano (2002) «L'européanisation des structures de représentation des intérêts », in Grossman, Emiliano ; Saurugger, Sabine (dir.) La Politique européenne, Paris, Centre d'études européennes de Science Po, Nº 7, p. 45.

Güven, Özcan (1988) AET'de ve Türkiye'de bankacllk sektörü : uyumla ilgili sorunlar [Le secteur bancaire à la CEE et en Turquie : Les problèmes relatif à l'harmonisation], T/88 12-120, Istanbul, TÜsİAD.

Human Rights Watch Organisation (1998) « Human rights developments in Turkey », World report.

Kabaalioğlu, Haluk (1989) AET'de şirket kanunu ve sermaye piyasalar : Türkiye açısından bir değerlendirme [Le code d'entreprise et les marchés du capital en CEE : une évaluation du point de vue de la Turquie], T/89 3-125, Istanbul, TÜsíAD.

Kaytaz, Mehmet ; Özmucur, Süleyman (1986) Dış Ticaret Raporu [Rapport sur le commerce extérieur], T/86 9-91, Istanbul, TUSIAD, pp. 41-67.

Keyder, Çağlar (2003) Memâlik-i Osmaniye'den Avrupa Birliğine [Des pays ottomans à l'Union européenne], Istanbul, İletişim, pp. 167-171.

Keyder, Çağlar (2004) Türkiye'de Devlet ve Sinıflar [État et classes en Turquie], Istanbul, İletişim, pp. 160-187.

Knill, Christoph ; Lehmkuhl, Dirk (1999) «How Europe Matters? Different Mechanisms of Europeanization», in European Integration online papers (EIoP), vol. 3, N ${ }^{\circ}$ 7, p. 3 http://eiop.or.at/ eiop/index.php/eiop, dernière consultation le 5 juin 2007.

Lagroye, Jacques (2003) « Le processus de politisation », in J. Lagroye (ed.) La politisation, Paris, Belin, pp. 362-366.

«Le sens de la responsabilité civique de Sakıp Sabancı », Türk İhracatçılar Meclisi - Turkishtime [publication de l'assemblée des exportateurs turcs], juin 2004.

Şener, Orhan (1988) AET ve Türkiye'de ulaşım sektörü : uyumla ilgili sorunlar [Le secteur des transports à la CEE et en Turquie : les problèmes relatifs à l'harmonisation], T/88 12-121, Istanbul, TÜSİAD.

Seufert, Günter (2002) « Milliyetçi Söylemlerin Sivil Toplum Üzerindeki Etkisi » [Les influences des discours nationalistes sur la société civile], in Institut français d'études anatoliens (IFEA) (dir.) Türkiye'de Sivil Toplum ve Milliyetçilik [Nationalisme et Société civile en Turquie], Istanbul, İletişim, p. 30 .

Tanör, Bülent (1997) Türkiye'de demokratikleşme Perspektifleri [Les perspectives de démocratisation en Turquie], T/96 204, Istanbul, TÜsİAD. 
TÜSİAD (1986), Guide to business and investment in Turkey [Guide des affaires et des investissements en Turquie].

TÜSİAD (1987) Türkiye'nin AET'ye üyeliği meselesi [La question de l'adhésion de la Turquie à la CEE], T/87 4-99, Istanbul, TÜsİAD.

TÜSİAD (1988) İspanya ve Portekiz'de ekonomik durum : AET'ye üye olduktan sonra [La situation économique en Espagne et au Portugal : après l'adhésion dans la CEE], T/88 4-114, Istanbul.

TÜSİAD (1992) Yeni Bir Anayasa için [Pour une nouvelle constitution], Istanbul, URL : http:// www.byegm.gov.tr/ayintarihidetay.aspx?Id=229\&Yil=1992\&Ay=3, dernière consultation le 24 juillet 2007.

TÜSİAD (2006) TUSIAD Yayınları [Les publications de TÜSİAD], İstanbul, pp. 4-5.

TÜSİAD Avrupa Birliği Temsilciliği (1997) Demokratikleşme raporu ve Avrupa [Rapport de démocratisation et l'Europe], Bruxelles.

\section{NOTES}

1. Les trois rapports traitant de la question de la démocratisation, qui ont été publiés entre 1997-2001, proposent des amendements constitutionnels et juridiques dans ces domaines.

2. Ce conseil est composé de 7 membres. Sa consultation est obligatoire avant la prise de décisions par le conseil d'administration. Ce dernier, composé de 11 membres, est également en charge de l'exécution des décisions. Le Haut Conseil consultatif n'a pas de rôle dans l'élaboration des décisions ni dans leur exécution.

3. TUSIAD (2009), Tanitım [Présentation], URL: http://www.tusiad.org/tusiad_cms.nsf/ TanitimTR.pdf

4. Lagroye, Jacques (2003) « Le processus de politisation », in J. Lagroye (ed.) La politisation, Paris, Belin, pp. 365-366.

5. Pour une définition de la politisation comme un processus de transgression de certaines règles sociales, voir ibid. p. 361.

6. Le journaliste turc Can Dündar est l'une des rares personnes à avoir accédé aux notes personnelles de Vehbi Koç. Can Dündar (2006) L'archive privée de Vehbi Koç a été ouverte à la $10^{e}$ anniversaire de son décès, URL : www.candundar.com.tr. Pour une lecture générale sur le contexte des relations entre les grands entrepreneurs turcs et les gouvernements du parti démocrate (DP [Demokrasi Partisi]) et du parti républicain du peuple (CHP [Cumhuriyet Halk Partisi]) entre 1950-1970, voir Buğra, Ayșe (1997) Devlet ve İşadamları [État et hommes d'affaires], İstanbul, İletişim, pp. 176-197 ; Keyder, Çağlar (2003) Memâlik-i Osmaniye'den Avrupa Birliğine [Des pays ottomans à l'Union européenne], İstanbul, İletişim, pp. 167-171; Keyder, Çağlar (2004) Türkiye'de Devlet ve Sinıflar [État et classes en Turquie], İstanbul, İletişim, pp. 160-187.

7. Avant tout, les membres devaient avoir un certain niveau de chiffres d'affaires et les petites et moyennes entreprises n'étaient pas représentées. Comme nous l'a expliqué Ümit İzmen, soussecrétaire général actuel en charge des affaires économiques, les candidats recevaient des questionnaires sur leurs tendances politiques et intellectuelles. Les réponses données étaient prises en compte par le conseil d'administration pour valider ou non l'adhésion (entretien avec Ümit İzmen, İstanbul, 3 août 2005).

8. Pour des informations plus détaillées sur cette campagne voir Ayşe Buğra, op. cit., p. 205.

9. Sur la méfiance des grands entrepreneurs turcs vis-à-vis des directeurs professionnels, voir Buğra, Ayşe, op. cit, p. 296-298.

10. « TÜSİAD’n yeni yüzü » [Le nouveau visage de TÜSİAD], Capital, 1 novembre 2001. 
11. Il s'agit d'un ancien ingénieur, ancien ministre, ancien bureaucrate, disposant de nombreuses connaissances et expériences sur l'industrie turque.

12. Le conseil d'administration formé par Kocatopçu a été le plus critiqué dans l'histoire de TÜSİAD. Cf. URL: http://ekonomitarihi.blogspot.com/2006/01/1985.html, consulté dernièrement le $1^{\mathrm{er}}$ avril 2009.

Pour les débats sur la composition du conseil d'administration en 1985, voir « TÜSİAD'in yeni yüzü ", [Le nouveau visage de TÜSİAD], Capital, $1^{\mathrm{er}}$ novembre 2001.

13. Né en 1933 et décédé en 2004, Sakıp Sabancı avait une position particulière au sein de TÜSİAD dès sa fondation. Propriétaire du deuxième plus grand groupe industriel de Turquie, il avait en outre une expérience dans la représentation des intérêts extérieurs à TÜSİAD en tant que président des Chambres d'Industrie d'Adana et de Kocaeli, et de l'Union des chambres d'industrie et de commerce de Turquie (entre 1964 et 1985).

14. Entretien avec Bülent Eczacıbaşı, ancien président de TÜSİAD et président du groupe Eczacıbaşı «Le sens de la responsabilité civique de Sakıp Sabanc1 », Turkishtime (publication de l'assemblée des exportateurs turcs [Türk İhracatçllar meclisi]), juin 2004.

15. Dans la définition qu'il donne de son système éducatif, le Robert College insiste sur des notions telles que l'« universalité », l'« ouverture ", l'« être communicatif », la «créativité », la «diversité ", la «pensée critique », la "capacité à devenir une élite, un leader en Turquie et même à l'étranger». Ainsi, malgré son rattachement officiel au ministère de l'Education nationale, il se présente comme un lycée qui se distingue par de l'approche officielle de l'éducation, unitariste, qui tend à imposer une formation en conformité avec l'idéologie turcoislamique. De même, le lycée allemand d'Istanbul a pour objectif la formation de générations proches de deux cultures, la culture germanophone (non seulement allemande, mais aussi suisse et autrichienne) et la culture turque. Ainsi, l'approche unitaire ne s'applique pas dans son programme d'enseignement. Cf. URL: http://www.ds-İstanbul.de/tuerkisch/main_tr.htm, consulté dernièrement le 23 mars 2007.

16. Les mesures portaient sur la dévaluation de la livre turque (pour l'achat des devises à leur vraie valeur), la diminution des coûts de production, la diminution des salaires, un régime d'importation de plus en plus libéral, les subventions et encouragements à l'exportation, l'encouragement des capitaux étrangers, l'orientation de la production des entreprises vers les marchés extérieurs. Cf. Korkut Boratav (2003) Türkiye İktisat Tarihi 1908-2002 [Histoire économique de la Turquie 1908-2002], İmge, Ankara, p. 149.

17. Alors qu'en 1980 ces pays absorbaient $22 \%$ des exportations industrielles turques, en 1982 ils en ont reçu $48 \%$, et leur part n'a jamais été inférieure à $42 \%$ jusqu'en 1986. Mehmet Kaytaz, Süleyman Özmucur, Dış Ticaret Raporu [Rapport sur le commerce extérieur], T/86 9-91, TÜsİAD, İstanbul, 1986, p. 41.

18. Kaytaz, Mehmet; Özmucur, Süleyman (1986), Dış Ticaret Raporu [Rapport sur le commerce extérieur], T/86 9-91, İstanbul, TUSIAD, pp. 41-67.

19. Entre 1980 et 1986, il n'y a pas eu de réunion de haut niveau entre les responsables politiques turcs et européens, et les institutions européennes avaient unilatéralement suspendu l'accord d'association jusqu'à ce que le régime politique turc respecte certains principes démocratiques. Sur la suspension des relations voir, «Résolution sur la peine de mort imposée à 52 leaders d'associations turques, le 22 janvier 1982 » in Türkiye Büyük Millet Meclisi (TBMM), Dışilişkiler ve Protokol Müdürlüğ̈̈ [Direction des relations extérieures et du protocole de la Grande Assemblée de Turquie], Les décisions prises par le Parlement européen à propos de la Turquie depuis 1980, Ankara, décembre 1980, p. 11.

20. TÜsİAD (1986), Guide to business and investment in Turkey [Guide des affaires et des investissements en Turquie]; TÜsİAD (1987) Türkiye'nin AET'ye üyeliği meselesi [La question de l'adhésion de la Turquie à la CEE], T/87 4-99, İstanbul. L'association a produit des analyses sur la situation économique de nouveaux membres de la CEE : TÜSİAD (1988) İspanya ve Portekiz'de 
ekonomik durum: AET'ye üye olduktan sonra [La situation économique en Espagne et au Portugal : après l'adhésion dans la CEE], T/88 4-114, İstanbul. Elle a aussi publié des rapports sur les questions d'harmonisation avec les normes européennes dans le système bancaire (Güven, Özcan [1988] AET'de ve Türkiye'de bankacıllk sektörü : uyumla ilgili sorunlar [Le secteur bancaire à la CEE et en Turquie: Les problèmes relatif à l'harmonisation], T/88 12-120, İstanbul), le secteur des transports (şener, Orhan [1988] AET ve Türkiye'de ulaşım sektörü : uyumla ilgili sorunlar [Le secteur des transports à la CEE et en Turquie : les problèmes relatifs à l'harmonisation], T/88 12-121, İstanbul), et le code de l'entreprise (Kabaalioğlu, Haluk [1989] AET'de şirket kanunu ve sermaye piyasalar: Türkiye açısından bir değerlendirme [Le code d'entreprise et les marchés du capital en CEE : une évaluation du point de vue de la Turquie], T/89 3-125, İstanbul).

21. Pour ces déclarations, voir Birand, Mehmet Ali (1996) Türkiye'nin Gümrük Birliği Macerast 1959-1996 [L'aventure d'union douanière de la Turquie 1959-1996], İstanbul, Ad yayıncılık, p. 397.

22. La Turquie a fait sa demande d'adhésion à la CEE le 14 avril 1987.

23. Grossman, Emiliano (2002) «L'européanisation des structures de représentation des intérêts ", in Grossman, Emiliano ; Saurugger, Sabine (eds.) in La Politique européenne, Paris, Centre d'études européennes de Science Po, $\mathrm{N}^{\circ} 7$, p. 45.

24. Voir Avis de la Commission sur la demande d'adhésion de la Turquie à la Communauté, 20 décembre 1989, SEC (89) 2290 final, http://www.ena.lu/mce.cfm, consulté le 2 août 2007.

25. Pour les effets des unions douanières sur les structures domestiques, voir Knill, Christoph ; Lehmkuhl, Dirk (1999) «How Europe Matters? Different Mechanisms of Europeanization", in European Integration online papers (EIoP), vol. 3, N ${ }^{\circ}$, p.3 http://eiop.or.at/eiop/index.php/eiop , dernière consultation le 2 Août 2007.

26. Ibid.

27. Eczacıbaşı, Bülent (1992), « Toplumun Katılımına Açık Bir Anayasa » [Une constitution ouverte à la participation de la société], in Görüş, İstanbul, TÜsİAD, pp. 15-16.

28. TÜSİAD (1992) Yeni Bir Anayasa için [Pour une nouvelle constitution], İstanbul. Le résumé du projet peut être consulté sur le site internet de la Direction générale de la presse et de l'information du Premier ministre,

URL : http://www.byegm.gov.tr/ayintarihidetay.aspx?Id=229\&Yil=1992\&Ay=3. Consulté le 24 juillet 2007.

29. Ces deux instituts fonctionnent dans le cadre de l'« Institut supérieur Atatürk de culture, de langue et d'histoire" crée par la Constitution de 1982. Ils ont pour mission "d'effectuer des recherches sur la pensée, les principes et les réformes d'Atatürk, ainsi que sur les domaines de la culture, de l'histoire, et de la langue de turques ; de les faire connaître, de les propager et d'éditer des publications à leur sujet ». Cf. Article 134, Constitution de la République de Turquie, Ankara, T.C Başbakanlı̆̆1, 1995, p. 74. Günter Seufert explique que ces instituts sont investis dans les programmes d'enseignements des écoles publiques et privées et dans la rédaction des livres scolaires en référence aux postulats de l'idéologie officielle reflétée dans la Constitution de 1982. Voir Seufert, Günter (2002) «Milliyetçi Söylemlerin Sivil Toplum Üzerindeki Etkisi » [Les influences des discours nationalistes sur la société civile], in Yerasimos, S.; Seufert, G.; Vorhoff, K. (eds) Türkiye'de Sivil Toplum ve Milliyetçilik [Nationalisme et Société civile en Turquie], İstanbul, İletişim, p. 30.

30. Selon la Commission européenne, depuis 1980 le régime politique turc s'était progressivement approché des régimes politiques des pays membres, grâce aux élections et au fonctionnement de la démocratie parlementaire. En revanche, la législation turque continuait à limiter les libertés associatives et encadrait strictement l'action collective. La Commission a également noté que « la situation des droits de l'homme et le respect de l'identité des minorités, quoique ayant fait l'objet d'une évolution au cours des dernières années, n'ont pas encore atteint le niveau nécessaire pour une démocratie ». Voir «Introduction », Avis de la Commission sur la 
demande d'adhésion de la Turquie à la Communauté, 20 décembre 1989, SEC (89) 2290 final, http://www.ena.lu/mce.cfm. Consulté le $1^{\mathrm{er}}$ avril 2007.

31. M. Halis Komili est un entrepreneur qui fait partie de la même génération que Ömer Dinçkök, Cem Boyner et Bülent Eczacıbaşı. Il est né en 1947 et, comme ses trois prédécesseurs, il est diplômé du Robert College (en 1969). Tout comme Ömer Dinçkök, il a poursuivi des études en ingénierie chimique à l'école supérieure du Robert College. Il est ensuite parti en Angleterre pour son master en gestion d'entreprise. Il est devenu membre de TÜSİAD en 1979 et a accédé au conseil d'administration de l'association en 1987, sous la présidence d'Ömer Dinçkök.

32. Entretien avec Hale Hatipoğlu, sous-secrétaire de TÜSİAD en charge du département des relations extérieures à la date de l'entretien, Istanbul, 14 avril 2003.

33. Entretien avec Sinan Ülgen, ancien diplomate au sein de la délégation permanente de Turquie auprès de l'Union européenne (période d'activité : 1993-1996), Istanbul, 4 août 2005.

34. Ibid.

35. Entretien avec Hale Hatipoğlu, op. cit.,14 avril 2003.

36. Entretien avec Adriaan Van der Meer, chef d'unité "Turquie » de la direction générale d'élargissement de l'UE à la date de l'entretien, Bruxelles, 12 avril 2002.

37. Les huit comités portaient respectivement sur: les affaires parlementaires; les affaires économiques et financières; les affaires extérieures; le secteur bancaire et les affaires industrielles; les affaires d'entreprise; les affaires sociales; la société d'information et les nouvelles technologies ; les relations avec les associations locales et sectorielles.

38. Entretien avec Ebru Dicle, sous-secrétaire responsable des affaires parlementaires à la date de l'entretien, 6 avril 2003, Istanbul.

39. L'acquis communautaire correspond au socle commun de droits et d'obligations qui lie l'ensemble des États membres au titre de l'Union européenne. Les pays candidats doivent accepter cet acquis communautaire avant d'adhérer à l'Union. Cf. Europa Glossaire, Acquis communautaire, http://europa.eu/scadplus/glossary/community_acquis_fr.htm. Consulté dernièrement le 30 septembre 2007.

40. Le secrétaire général est composé de salariés professionnels à plein temps. Il gère les activités quotidiennes des départements et assure la permanence et la mémoire de l'association.

41. Avant 1995, signalons l'exception de 1987 où 23 études ont été rédigées sous l'effet conjugué de la demande d'adhésion à la CEE, du changement de direction et de la montée en puissance de la nouvelle génération. Mais l'accroissement ne s'est pas maintenu : en 1988, ce chiffre est tombé à quatorze, et en 1989 à neuf rapports. Cf. TÜSİAD (2006) TUSIAD Yayınları [Les publications de TÜSİAD], İstanbul, TÜSİAD, pp. 4-5.

42. Tanör, Bülent (1997) Türkiye'de demokratikleşme Perspektifleri [Les perspectives de démocratisation en Turquie], T/96 204, İstanbul, TÜsİAD.

43. Lagroye, Jacques (2003) op. cit., p. 366.

44. TÜSİAD, « Publications », in TÜSİAD Brochure (version française), www.tusiad.org. Consulté le 30 Septembre 2007.

45. Selon Jacques Lagroye, ce sont des actions exemplaires qui ont une portée politique générale, c'est-à-dire que leurs auteurs dépassent les limites de leur légitimité politique et s'engagent dans des aspects de la vie politique qui ne concernent pas directement leur fonction sociale, mais le champ politique dans sa globalité. Cf. Lagroye, Jacques, op. cit., pp. 362-363.

46. Entretien avec Ümit İzmen, op. cit., Istanbul , 3 août 2005.

47. Président du comité des affaires parlementaires de TÜSİAD depuis qu'il a été fondé en 1995. Can Paker est aussi le directeur de TESEV, Türkiye Ekonomik ve Sosyal Etüdler Vakfi [Fondation des études économiques et sociales de Turquie], depuis sa fondation en 1994. Il a été une figure très médiatique en Turquie au début de la décennie 2000.

48. Diplômé du Robert College, Erkut Yucaoğlu a obtenu un doctorat en ingénierie industrielle à Stanford en 1973. Il a été président du conseil d'administration de TÜSİAD entre 1999 et 2000, 
puis vice-président du High Advisory Council de TÜSİAD. Erkut Yücaoğlu entretient de bonnes relations avec l'armée. Pour des informations sur ses rencontres avec certains responsables militaires pendant sa présidence, voir Milliyet, 6 juillet 1998 ; Hürriyet, 4 décembre 1998 ; Milliyet, 19 juillet 1999.

49. Entretien avec Serkan Ersöz, responsable au sein du département des affaires parlémentaires de TÜSIAD à la date de l'entretien, 6 avril 2003, Istanbul.

50. Par exemple, Human Rights Watch Organisation (1998), «Human rights developments in Turkey ", in World report.

51. Michael Lake, représentant de la Commission européenne en Turquie entre 1991-1998, relevait que la Turquie pourrait instantanément devenir un membre de l'UE si tout ce qui était proposé dans ce rapport était mis en œuvre. Cf. TÜSİAD, Avrupa Birliği Temsilciliği (1997), Demokratikleşme raporu ve Avrupa [Rapport de démocratisation et l'Europe], Bruxelles.

52. Entretien avec Adriaan Van der Meer, op cit., Bruxelles, 12 avril 2002.

53. Entretien avec Sinan Ülgen, op. cit, İstanbul, 4 août 2005.

54. Enretien avec Aldo Kaslowski, vice-président de TÜSIAD à la date de l'entretien et président de TÜSIAD International depuis sa création en 2001, 17 avril 2004, İstanbul.

55. Entretien avec Aldo Kaslowski, ibid.

56. Entretien avec Serkan Ersöz, op. cit., 6 avril 2003, Istanbul.

\section{RÉSUMÉS}

Since the second half of the 1990s, Turkish political life has been markedly influenced by the growing importance of TÜSİAD, an entrepreneurs' association which calls for a transformation of the political regime/political system. The association advocates a new, more democratic political system, referring to the reforms expected by the European institutions in the course of / as part of the EU integration process of Turkey. This situation is at odds with the initial goals of the association. TÜSIAD was founded by leading entrepreneurs with the aim of promoting "free entrepreneurship" in Turkey, in a situation where the founding membres felt "threatened" by the risk of an anti-liberal economy and/or a social environnement within which their businesses were not respected. This article analyses how this association, which originally aimed at promoting free-entrepreneurship and market economy, ended up upholding political and legal reforms aiming at the europeanization of the Turkish political system - and consequently, of Turkey.

\section{INDEX}

Mots-clés : association, entreprises, européanisation, Turquie-Europe, lobbying, TÜSIAD

Keywords : Europeanization, lobbying, Turkey-Europe, TÜSIAD 


\section{AUTEUR}

\section{OZAN SERDAROGLU}

Dr. Ozan Serdaroğlu received his MA and Ph.D degrees from "Institut d'Études politiques" of Aixen-Provence. Before joining the ISDP he was working as Assistant Professor and Director of Vocational School at the "European University of Lefke". He has also been affiliated with "Institut de recherches et d'études sur le monde arabe et musulman", UNESCO and the European Commission. 